\title{
Numerical Modeling of Gas Flows in the Transition between Rarefied and Continuum Regimes ${ }^{\ddagger}$
}

\author{
M. Günther*, P. Le Tallec **, J.P. Perlat ${ }^{* *}$, J. Struckmeier* \\ *University of Kaiserslautern ${ }^{* *}$ INRIA \\ Department of Mathematics Domaine de Voluceau \\ Rocquencourt \\ P.O.Box 3049 \\ Boite Postale 105 \\ D 67653 Kaiserslautern \\ F 78153 Le Chesnay Cedex
}

\section{Introduction}

The Boltzmann equation is an appropriate model to simulate rarefied gas flows as long as the mean free path of the gas is not too small. However, in the transition from rarefied to continuum flows, numerical simulations of the Boltzmann equation reach the limits of the existing hardware platforms, even when using massively-parallel systems.

Hence, it is worthwhile to investigate the transition from the Boltzmann equation to classical continuum mechanics, like Eulcr or Navier-Stokes equations and to combine both models in terms of a domain decomposition approach. Besides others, one of the challenging problems applying a domain decomposition procedure, is to detect (automatically) those regions, where it is possible to switch to the more simplified fluid dynamic models as well as to formulate appropriate coupling conditions between the different flow models. Recently, several authors investigated various domain decomposition methods based on kinetic and continuum flow models, like the formulation of coupling conditions as well as the derivation of criteria to automatically detect kinetic and continuum regimes within the gas flow $[1,6,9,10,11,12,17,19]$.

The aim of the present investigation is twofolded: the first aim is to generalize the existing domain decomposition methods to the case of a rarefied gas with real gas effects, i.e. generalized Boltzmann equations, which include rotational and vibrational degrees of freedom as well as chemical reactions in the gas. Moreover, we like to present a new approach based on the fourteen moments expansion of Levermore [13], which combines a Galerkin approximation for the Boltzmann equation with a particular expansion of the solution of the Boltzmann equation, which is in contrast to the well-known Chapman-Enskog

\footnotetext{
₹o appear in "Notes on Numerical Fluid Mechanics", 1998
} 
expansion strictly positive. In particular, we investigate how the resulting equations may degenerate to a more standard Navier-Stokes model.

The paper is organized as follows: in the next section we present briefly the generalized Boltzmann equation based on diatomic molecules, which include energy exchange between translational, rotational and vibrational degrees of freedom. At the end of the section we propose a simplified model problem, which is used in Section 3 to perform an asymptotic analysis to derive the limiting fluid dynamic equations for the Boltzmann equation with internal degrees of freedom. In Section 4 we show, how the Navier-Stokes equations may be obtained from the 14-moments expansion of Levermore using the well-known Hilbert expansion technique. Moreover, the somewhat original derivation clearly emphasize a kinetic interpretation of the moment expansion used by Levermore and we discuss the problem how to define a coupling process between the two models and give numerical simulations to validate the proposed coupling strategy.

\section{The Boltzmann Equation for Diatomic Molecules}

\subsection{The Description of Internal Degrees of Freedom}

In the following we consider a rarefied gas consisting of identical diatomic molecules, where each molecule carries besides the translational energy two additional degrees of freedom, namely a rotational and vibrational energy.

Due to the small level distance for rotational energy states, it is appropriate to simplify the resulting model considering the rotational energy as a continuous variable $e \in \mathbb{R}_{0}^{+}$, whereas the vibrational energy is assumed to take only discrete values $\nu_{i} \in \mathcal{D}$, where $\mathcal{D}$ denotes the finite set of different vibrational levels.

Then, the generalized Boltzmann equation for diatomic molecules describes the time evolution of a kinetic density function $f\left(x, v, e, \nu_{i}\right)$ - the phase space density - in the form

$$
\partial_{t} f+v \nabla f=\frac{1}{\varepsilon} Q(f, f)
$$

where $Q(f, f)$ denotes the collision operator given by

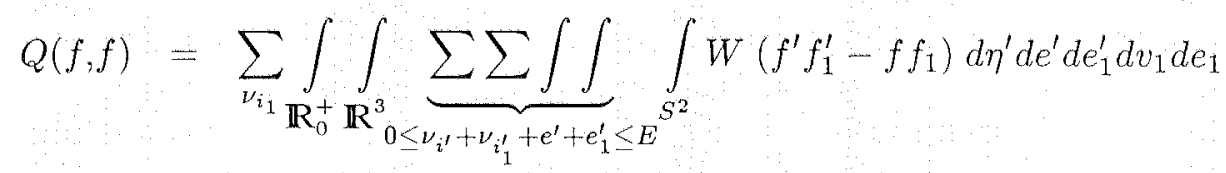

and $f^{\prime}=f\left(x, v^{\prime}, e^{\prime}, \nu_{i^{\prime}}\right), f_{1}^{\prime}=f\left(x, v_{1}^{\prime}, e_{1}^{\prime}, \nu_{i_{1}^{\prime}}\right)$ etc. The collisions scattering kernel $W$ (the transition probability) is assumed to take the form 


$$
W=\left|v-v_{1}\right| \sigma\left(E ; \eta, \nu_{i}, \nu_{i_{1}}, e_{;} e_{1} \rightarrow \eta^{\prime}, \nu_{i^{\prime}}, \nu_{i_{1}^{\prime}}, e^{\prime}, e_{1}^{\prime}\right),
$$

where the function $\sigma$ denotes the differential cross section, $E$ the total collision energy given by $E=\frac{1}{4}\left|v-v_{1}\right|^{2}+e+e_{1}+\nu_{i}+\nu_{i_{1}}$ and $\eta=\left(v-v_{1}\right) /\left|v-v_{1}\right|$. In (2.1) we already assumed a hydrodynamic scaling $x \rightarrow \varepsilon x, t \rightarrow \varepsilon t$ to rolate the time evolution of the rarefied gas with the so-called Knudsen number IKn $-\lambda / L$, where $\lambda$ denotes the mean free path of the gas, $L$ a characteristic length of the problem, respectively.

An essential part in the generalized Boltzmann equation is to define appropriate, but in the same way simple models to specify the differential cross section $\sigma$. In the following, we use a differential cross section based on the generalized Borgnakke-Larsen model [18], which can be written in the form

$$
\sigma_{g b l}=(1-a(E)-b(E)) \sigma_{e l}+a(E) \sigma_{v e}+b(E) \sigma_{i n},
$$

where $0 \leq a(E), b(E)$ and $a(E)+b(E) \leq 1$ for all $E \in \mathbb{R}_{0}^{+}$and

$$
\begin{aligned}
\sigma_{e l} & =\varphi\left(\eta \cdot \eta^{\prime}\right) \sigma_{g b b}^{t o t}(E) \delta\left(e-e^{\prime}\right) \delta\left(e_{1}-e_{1}^{\prime}\right) \delta_{i i^{\prime}} \delta_{i_{1} i_{1}^{\prime}} \\
\sigma_{v e} & =\frac{6}{\left(E-\nu_{i}-\nu_{i_{1}}\right)^{3}} \varphi\left(\eta \cdot \eta^{\prime}\right) \sigma_{g b l}^{t o t}(E)\left(E-e^{\prime}-e_{1}^{\prime}-\nu_{i^{\prime}}-\nu_{i_{1}^{\prime}}\right) \delta_{i i^{\prime}} \delta_{i_{1} i_{1}^{\prime}} \\
\sigma_{i n} & =\frac{6}{\sum_{\nu_{i^{\prime}}+\nu_{i_{1}^{\prime}} \leq E}\left(E-\nu_{i^{\prime}}-\nu_{i_{1}^{\prime}}\right)^{3}} \varphi\left(\eta \cdot \eta^{\prime}\right) \sigma_{g b l}^{t o t}(E)\left(E-e^{\prime}-e_{1}^{\prime}-\nu_{i^{\prime}}-\nu_{i_{1}^{\prime}}\right)
\end{aligned}
$$

Moreover, we assume isotropic scattering for the angular dependence in the collisions, i.e.

$$
\varphi\left(\eta \cdot \eta^{\prime}\right)=\frac{1}{4 \pi}
$$

In the Borgnakke-Larsen-type models [8], one assumes a differential cross section, which is a combination of elastic, vibrational elastic and completely inelastic parts, i.e. in the first case we have no energy exchange between translation and internal energies, in the second case the vibrational energy is preserved and in the third case we have full energy exchange between ail degrees of freedom. Hence, even the collision operator can be written as a sum of three different operators, $Q=Q_{e l}+Q_{v e}+Q_{i n}$. The functions $a(E), b(E)$ in (2.2) may be determined using macroscopic models [18], e.g. the Parker model for rotational relaxation determines the function $a(E), b(E)$ may be fixed assuming a Millikan-White model for vibrational relaxation, where both models contain some parameters, which are fitted to experimental data. 


\subsection{Definition of a Model Problem}

In particular at high temperatures, the relaxation times for translational, rotational and vibrational energies may differ strongly and this may influence the resulting fluid dynamic limits of the generalized Boltzmann equation. Here, one may expect to obtain modified Navier-Stokes equations, which include additional temperature equations for the rotational as well as vibrational states of molecules.

In the following section we will study the fluid dynamic limits of a simplified model problem, where the relaxation times between translational and rotational energies differ by some parameter $\delta$, the vibrational states are kept frozen. Moreover, we restrict ourselve to a one-dimensional steady-state problem in a slab, with diffusive boundary conditions based on the two wall temperatures $T_{L}$ and $T_{R}$ on the left and right wall of the slab, respectively.

To validate the asymptotic expansions obtained from the generalized Boltzmann equation, the model problem has been treated numerically applying a particle method to simulate rarefied gas flows [16].

Macroscopic quantities like the density or the mean velocity are defined as moments of the density function $f(t, x, v, e)$, like

$$
\begin{aligned}
\text { mass density }: \rho(t, x) & =\langle f\rangle_{v, e} \\
\text { mean velocity }: u(t, x) & =\frac{1}{\rho}\langle v f\rangle_{v, e} \\
\text { (kinetic) temperature : } T(t, x) & =\frac{2}{3} \frac{1}{\rho}\left\langle c^{2} f\right\rangle_{v, e} \\
\text { rotational temperature : } T_{r o t}(t, x) & =\frac{1}{\rho}\langle e f\rangle_{v, e} \\
\text { stress tensor : } \sigma(t, x) & =-\langle c \otimes c f\rangle_{v, e} \\
\text { heat flux vector : } q(t, x) & =\left\langle\frac{1}{2} c^{2} c f\right\rangle_{v, e}
\end{aligned}
$$

where $\langle\cdot\rangle_{v, e}$ denotes the integration over $v$ and $e$ and $c=v-u$.

\section{Asymptotic Expansions}

In the following we consider the steady-state, one-dimensional Boltzmann equation in a slab written in the form

$$
v_{x} \partial_{x} f=\frac{1}{c} Q_{e l}(f, f)+\frac{\delta}{c} Q_{v e}(f, f), \quad x \in[0,1] .
$$


where we assume, that the vibrational states of molecules are kept frozen and $\delta$ denotes a parameter to relate the translational and rotational relaxation times. In particular, we study the asymptotic limits as $\varepsilon, \delta \rightarrow 0$ to obtain macroscopic equations for the density $\rho$ and the temperatures $T$ and $T_{r o t}$, the kinetic and rotational temperature of the gas. This is achieved using an asymptotic expansion for $f(t, x, v, e)$ and the most general Ansatz would be a double expansion in terms of $\varepsilon$ and $\delta$ in the form

$$
f=\sum_{k, l=0}^{\infty} \varepsilon^{k} \delta^{l} f_{\delta}^{(k, l)}
$$

A more simple analysis is to study the asymptotic limits only along particular traces $(\varepsilon, \delta) \rightarrow 0$, i.e. to assume a functional dependence between $\varepsilon$ and $\delta$ in the form $\delta=\delta(\varepsilon)$. Then, the double expansion is reduced to a single expansion with respect to the small parameter $\varepsilon$ given by

$$
f(x, v, e)=\sum_{j=0}^{\infty} \varepsilon^{j} f^{(j)}(x, v, e)
$$

For the single functions $f^{(j)}, j \geq 1$ we demand the normalization condition

$$
\left\langle\Psi(v, e) f^{(j)}\right\rangle_{v, e}=0
$$

where $\Psi(v, e)=\left(1, v, v^{2}, k(e)\right)^{t}, k(e)$ denotes an arbitrary (non-constant) function in e. Condition (3.3) implies, that the macroscopic quantities $\rho, u, T$ and $T_{\text {rot }}$ are completely determined by $f^{(0)}$. Moreover, we use the (formal) decomposition $f^{(j)}(x, v, e)=h^{(j)}(x, v, e) g(x, e)$ for $j \geq 0$.

\subsection{The Case $\delta=0$}

In this case the Boltzmann equation can be rewritten as

$$
v_{x} \partial_{x} f=\frac{1}{\varepsilon} Q_{e l}(f, f)
$$

Inserting (3.2) into (3.4) yields

$$
\begin{aligned}
& \varepsilon^{-1}: Q_{e l}\left(f^{(0)}, f^{(0)}\right)=0 \\
& \varepsilon^{0}: \quad v_{x} \partial_{x} f^{(0)}=Q_{e l}\left(f^{(1)}, f^{(0)}\right) \\
& \varepsilon^{1}: \quad v_{x} \partial_{x} f^{(1)}=Q_{e l}\left(f^{(2)}, f^{(0)}\right)+Q_{e l}\left(f^{(1)}, f^{(1)}\right)
\end{aligned}
$$

From the equation at the $e^{-1}$-level we can write 


$$
h^{(0)}(x, v)=M_{\rho, u, T}(v)
$$

where $M_{\rho, u, T}(v)=\frac{\rho}{(\pi T)^{3 / 2}} \exp \left(-(v-u)^{2} / T\right)$ is a Maxwellian, $f^{(0)}=h^{(0)} g$ and the function $g(x, e)$ remains unknown at this level - up to the normalization condition

$$
\langle g\rangle_{e}=1 \text {. }
$$

Moreover, the velocity $u$ is zero, because integrating (3.4) with respect to $v$ and $e$ yields

$$
\partial_{x}(\rho u)=0 \Rightarrow \rho u=c
$$

and due to the diffusive boundary conditions, one has $(\rho u)(0)=(\rho u)(1)=0$, i.e. $c=0$. Since $\rho>0$, it follows $u=0$.

From the $\varepsilon^{0}$-level we obtain

$$
v_{x} g \partial_{x} h^{(0)}+v_{x} h^{(0)} \partial_{x} g=Q_{e l}\left(h^{(1)} g, h^{(0)} g\right)
$$

Now we split the function $h^{(1)}$ into two parts, namely $h^{(1)}(x, v, e)=h_{1}^{(1)}(x, v)+$ $h_{2}^{(1)}(x, v, e)$, to get a better representation of the first approximation and obtain from the $\varepsilon^{0}$-level the two equations

$$
\begin{aligned}
& v_{x} g \partial_{x} h^{(0)}=Q_{e l}\left(h_{1}^{(1)} g, h^{(0)} g\right) \\
& v_{x} h^{(0)} \partial_{x} g=Q_{e l}\left(h_{2}^{(1)} g, h^{(0)} g\right)
\end{aligned}
$$

We demand, that $h_{1}^{(1)} g$ and $h_{2}^{(1)} g$ fulfill the condition (3.3).

Eqs. (3.6), (3.7) are conditions for the unknown functions $h_{1}^{(1)}$ and $h_{2}^{(2)}$. The right side can be regarded as a linear operator for $h_{1}^{(1)}, h_{2}^{(1)}$ and the kernel for $L(\cdot)=Q_{e l}\left(\cdot h^{(0)} g, h^{(0)} g\right)$ is given by ker $L=\left\langle 1, v, v^{2}, k(e)\right\rangle$, where $k(e)$ is a non-constant function.

Then, (3.6) and (3.7) are solvable, if the conditions

$$
\begin{aligned}
& \left\langle\Psi v_{x} g \partial_{x} h^{(0)}\right\rangle_{v, e}=0 \\
& \left\langle\Psi v_{x} h^{(0)} \partial_{x} g\right\rangle_{v, e}=0
\end{aligned}
$$

with $\Psi(v, e)=\left(1, v, v^{2}, k(e)\right)^{t}$ are fulfilled.

From (3.8) one gets

$$
\partial_{x}(\rho T)=0
$$

All other equations are identically equal to zero.

Then, the first order approximation is given by 


$$
h_{1}^{(1)}(x, v)=v_{x} \partial_{x} \ln T(x)\left(\frac{v^{2}}{T}-\frac{5}{2}\right) \Theta_{\rho, T}(x) h^{(0)}(x, v)+\left(b_{y} v_{y}+b_{z} v_{z}\right) h^{(0)}(x, v)
$$

If we use a collision model, such that the viscosity $\nu \sim T^{p}$, we obtain $\Theta_{\rho, T} \sim \frac{T^{p-1}}{\rho}$. In the case of Maxwellian molecules and rigid elastic spherical molecules we have $p=1$ and $p=0.5$, respectively.

The $\varepsilon^{1}$-order equation determines the function $f^{(2)}$ : the solvability equations are

$$
\left\langle\Psi\left(v_{x} \partial_{x}\left(h_{1}^{(1)} g+h_{2}^{(1)} g\right)-Q_{e l}\left(h_{1}^{(1)} g+h_{2}^{(1)} g, h_{1}^{(1)} g+h_{2}^{(1)} g\right)\right)\right\rangle_{v, e}=0
$$

From (3.11) we obtain

$$
\begin{aligned}
\partial_{x}\left(\lambda(\rho, T) \partial_{x} T\right) & =0 \\
\left\langle k(e) v_{x} \partial_{x} f^{(1)}\right\rangle_{v ; e} & =0
\end{aligned}
$$

where

$$
\lambda(\rho, T)=\left\langle v^{2} \frac{v_{a}^{2}}{T}\left(\frac{v^{2}}{T}-\frac{5}{2}\right) \Theta_{\rho, T} h_{\rho, T}^{(0)}(v)\right\rangle_{v}
$$

is the heat conduction coefficient. Again, all other equations are vanishing trivially.

Eqs. (3.10) and (3.12) determine the parameters $\rho$ and $T$. For example for the special model $\nu \sim T^{p}$, the solution is given by

$$
\begin{array}{ll}
\rho(x)=\frac{p_{0}}{\left(\left(T_{R}^{q}-T_{L}^{q}\right) x+T_{L}^{q}\right)^{1 / q}}, & p_{0} \in \mathbb{R}_{0}^{+} \quad x \in[0,1] \\
T(x)=\left(\left(T_{R}^{q}-T_{L}^{q}\right) x+T_{L}^{q}\right)^{1 / q}, & x \in[0,1],
\end{array}
$$

where $q=p+1$, i.e. we have $q=2$ for Maxwellian molecules and $q=1.5$ for rigid spherical molecules.

Eqs. (3.10) and (3.12) represent the standard Navier-Stokes equations for the one-dimensional heat transfer, in particular we obtain a diffusion equation for the temperature $T$, which defines the translational temperature of the gas. Moreover, the heat conduction coefficient $\lambda$ coincides with the expression obtained for a monoatomic gas.

However, we derived some more equations: for each $k(e)$ we obtain an additional equation (3.13); but, since we do not have an analytical expression for $f^{(1)}$, it is not possible to write the equation in terms of macroscopic quantities together with transport coefficients.

To verify our result, we performed numerical simulations using a particle method like discussed in Ref. [16]: Here, we used Maxwellian molecules at a Knudsen number $K n=0.01$ and wall temperatures $T_{L}=1$ and $T_{R}=2$. 

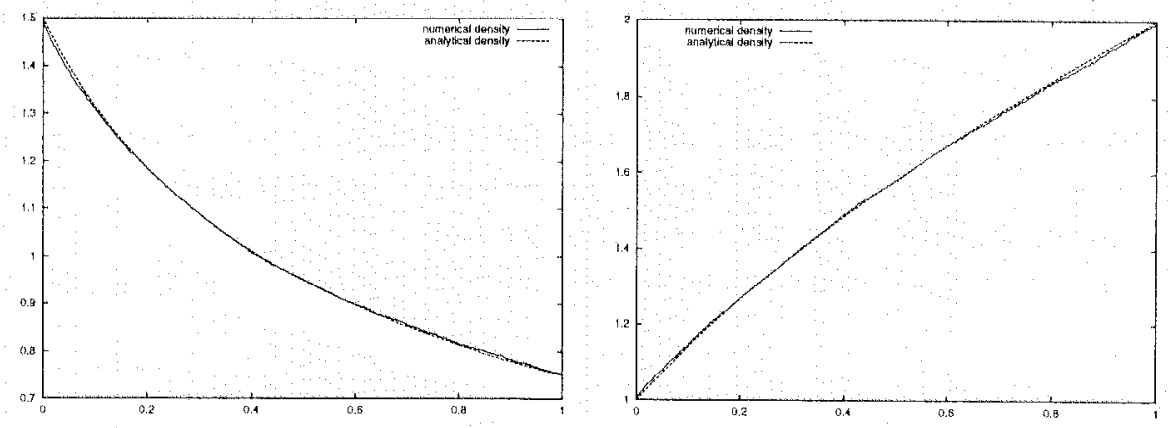

Figure 1 Density and Temperature Profile along the Slab

Fig. 1 shows the density and temperature profiles along the slab. The numerical results coincide nearly with the theoretical ones, which confirms the correctness of our derivation.

\subsection{Representation of the Density g}

It remains to find a representation for the internal energy density $g$. Since the internal energies do not influence the transport and collisions of the particles, the distribution should be a superposition of the internal boundary distributions. Therefore, we make the Ansatz

$$
g(x, e)=\beta(x) g_{L}(e)+\alpha(x) g_{R}(e),
$$

where $g_{L}$ and $g_{R}$ are the boundary conditions for the internal energy at $x=0$ and $x=1$, respectively. Condition (3.5) implies that $\beta(x)=1-\alpha(x)$ and the next step is to determine the function $\alpha(x)$ : we separate the gas into two species, where the first one carries the information of the internal energy of the left boundary and the second one of the right boundary. The density of these two species are denoted by $f^{L}$ and $f^{R}$, respectively. Then, the function $\alpha$ gives the mixing ratio, i.e. $\alpha=\rho^{R} / \rho$ and $1-\alpha=\rho^{L} / \rho$, where $\rho^{L}, \rho^{R}$ are the mass densities and $\rho=\rho^{L}+\rho^{R}$.

For the functions $f^{L}, f^{R}$ we have the system

$$
\begin{aligned}
& v_{x} \partial_{x} f^{L}=\frac{1}{\varepsilon}\left(Q\left(f^{L}, f^{L}\right)+Q\left(f^{L}, f^{R}\right)\right) \\
& v_{x} \partial_{x} f^{R}=\frac{1}{\varepsilon}\left(Q\left(f^{L}, f^{R}\right)+Q\left(f^{R}, f^{R}\right)\right)
\end{aligned}
$$

where $Q$ now denotes the standard monoatomic collision operator. For each density $f^{L}, f^{R}$ we use an asymptotic expansion in the form 


$$
f^{L}=\sum_{j=0}^{\infty} \varepsilon^{j} f_{L}^{(j)}, \quad f^{R}=\sum_{j=0}^{\infty} \varepsilon^{j} f_{R}^{(j)}
$$

Following $[3,4]$, we can write an approximation for $f^{L}, f^{R}$ in the form

$$
\begin{aligned}
f^{L}= & f_{L}^{(0)}+f_{L}^{(1)}=f_{L}^{(0)}\left(1+\Phi_{L}\right) \\
\Phi_{L}= & a_{1}\left(\frac{v^{2}}{T}-\frac{5}{2}\right) \frac{v_{x}}{\sqrt{T}} \partial_{x} \ln T \\
& \quad+d_{0} a_{0}^{f^{L}} \frac{v_{x}}{\sqrt{T}} d_{12}+d_{-1}\left(\frac{v^{2}}{T}-\frac{5}{2}\right) \frac{v_{x}}{\sqrt{T}} d_{12} \\
f^{R}= & f_{R}^{(0)}+f_{R}^{(1)}=f_{R}^{(0)}\left(1+\Phi_{R}\right) \\
\Phi_{R}= & a_{1}\left(\frac{v^{2}}{T^{\prime}}-\frac{5}{2}\right) \frac{v_{x}}{\sqrt{T}} \partial_{x} \ln T \\
& +d_{0} a_{0}^{f^{R}} \frac{v_{x}}{\sqrt{T}} d_{21}+d_{1}\left(\frac{v^{2}}{T}-\frac{5}{2}\right) \frac{v_{x}}{\sqrt{T}} d_{21}
\end{aligned}
$$

where $f_{L}^{(0)}=(1-\alpha) M_{\rho, 0, T}(v), f_{R}^{(0)}=\alpha M_{\rho, 0, T}(v)$ and all coefficients $a_{k}, d_{k}$, $k=-1,0,1, d_{12}, d_{21}$ depend on $\rho$ and $T$ (for the exact form see [3]).

For the mass fluxes we have the conditions

$$
\begin{aligned}
& q_{f^{L}}=\left\langle v f^{L}\right\rangle_{v}=\text { const } \\
& q_{f^{R}}=\left\langle v f^{R}\right\rangle_{v}=\text { const }
\end{aligned}
$$

With the approximation (3.17), we obtain for the mass flux $q_{f^{R}}$

$$
q_{f} \sim C(T) \partial_{x} \alpha
$$

where $C(T)$ depends on the collision model. For the hard sphere model we have $C(T) \sim T^{1 / 2}$ and in the case of Maxwellian molecules it holds $C(T) \sim T$. Condition (3.19) together with (3.20) yields $\alpha=c_{1} T+c_{2}$ and applying the boundary conditions we obtain

$$
\alpha(x)=\frac{T(x)-T_{L}}{T_{R}-T_{T}}
$$

If $T_{L}=T_{R}$, we can perform the limit $T_{L} \rightarrow T_{R}$ to get $\alpha(x)=x$. If $\alpha$ is determined, the resulting density $g$ has the form

$$
g=\frac{T_{R}-T}{T_{R}-T_{L}} g_{L}+\frac{T-T_{L}}{T_{R}-T_{L}} g_{R}
$$


To validate the expression for the density $g$ as given in Eq. (3.21), we performed some numerical experiments assuming diffusive boundary condition for the internal energy, i.e.

$$
g_{L}(e)=\frac{1}{T_{L, i}} \exp \left(-\frac{e}{T_{L, i}}\right), \quad g_{R}(e)=\frac{1}{T_{R, i}} \exp \left(-\frac{e}{T_{R, i}}\right) .
$$

The first set of simulations have been realized with Maxwellian molecules at a Knudsen number $K n=0.002$ using about $1.2 \cdot 10^{5}$ particles and boundary temperatures $T_{L}=1, T_{R}=2$ and $T_{L, i}=3, T_{R, i}=4$. The computational results obtained for the first 4 moments of the density function showed a quite good agreement between the numerical and analytical result. A further simulation was done, which should verify the correctness of the computation of $\alpha$ under the assumption (3.16) for the density $g$. In this case we use boundary conditions of the type

$$
g_{L}(e)=\delta\left(e-e_{L}\right), \quad g_{R}(e)=\delta\left(e-e_{R}\right), \quad \text { where } e_{L} \neq e_{R} .
$$

and the kinetic temperatures at the walls are $T_{L}=1, T_{R}=2$. The computations were done with Maxwellian molecules at Knudsen number $K n=0.01$ using 25.600 particles. Fig. 2 shows the fraction of particles, which have the internal energy $e_{R}$, where this number is equal to the function $\alpha$.

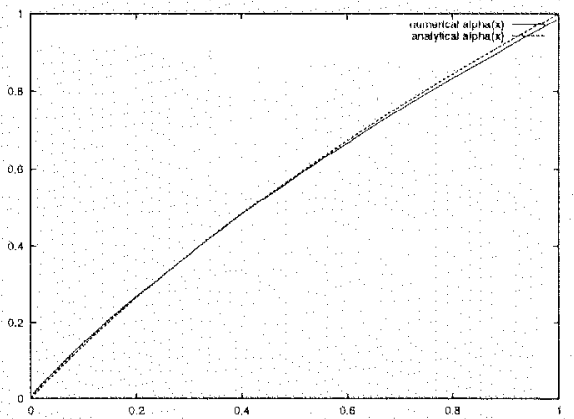

Figure 2 Comparison of Analytical and Numerical Results for $\alpha(x)$

All the simulations indicate, that the representation of $\alpha$ seems to be correct. The errors between the curves is explained by the numcrical inaccuracy. The curves do not differ much, such that we can regard them as a confirmation of our assumption for the function $g$.

\subsection{The Case $\delta=\varepsilon$}

Taking $\delta=\varepsilon$, we have the additional collision term $Q_{v e}(f, f)$ on the right hand side of (3.4) and the first equations in $\varepsilon$ now read 


$$
\begin{array}{ll}
\varepsilon^{-1}: & Q_{e l}\left(f^{(0)}, f^{(0)}\right)=0 \\
\varepsilon^{0}: & v_{x} \partial_{x} f^{(0)}=Q_{e l}\left(f^{(1)}, f^{(0)}\right)+Q_{v e}\left(f^{(0)}, f^{(0)}\right) \\
\varepsilon^{1} \quad: \quad & v_{x} \partial_{x} f^{(1)}=Q_{e l}\left(f^{(2)}, f^{(0)}\right)+Q_{e l}\left(f^{(1)}, f^{(1)}\right)+Q_{v e}\left(f^{(1)}, f^{(0)}\right)
\end{array}
$$

The solvability conditions for $f^{(1)}$ yield

$$
\begin{array}{cl}
0=\left\langle Q_{v e}\left(f^{(0)}, f^{(0)}\right)\right\rangle_{v, e} & \partial_{x}(\rho T)=0 \\
0=\left\langle v^{2} Q_{v e}\left(f^{(0)}, f^{(0)}\right)\right\rangle_{v, e} & 0=\left\langle k(e) Q_{v e}\left(f^{(0)}, f^{(0)}\right)\right\rangle_{v, e}
\end{array}
$$

If we define $J(g)(e)=\left\langle Q_{v c}\left(h^{(0)} g, h^{(0)} g\right)\right\rangle_{v}$, we can write

$$
0=\int_{\mathbb{R}_{0}^{+}}\left(\begin{array}{c}
1 \\
k(e)
\end{array}\right) J(g) d e
$$

which means, that the functions $1, k(e)$ are orthogonal to $J(g)$. If we choose a basis $b_{i}$, s.t. $\left\langle b_{i} b_{j}\right\rangle_{c}=\delta_{i j}$, then $J(g) \perp b_{i}$ for all $i$. Moreover, if the basis $b_{i}$ is complete, then $J(g)=0$, i.e. $g \in$ ker $J$, but we obtain no more information on $g$.

One can easily show, that the Maxwellian is contained in ker $J$ and numerical experiments indeed indicate, that the function $g$ is a Maxwellian. The solvability conditions for $f^{(2)}$ yield

$$
\begin{aligned}
0 & \left.=\left\langle Q_{v e}\left(h^{(1)} g, h^{(0)} g\right)\right)\right\rangle_{v, e} \\
0 & \left.=\left\langle v Q_{v e}\left(h^{(1)} g, h^{(0)} g\right)\right)\right\rangle_{v, e} \\
\partial_{x}\left(\lambda(\rho, T) \partial_{x} T\right) & =\left\langle v^{2} Q_{v e}\left(h^{(1)} g, h^{(0)} g\right)\right\rangle_{v, e} \\
\left\langle k(e) v_{x} \partial_{x} f^{(1)}\right\rangle_{v, e} & =\left\langle k(e) Q_{v e}\left(h^{(1)} g, h^{(0)} g\right)\right\rangle_{v, e}
\end{aligned}
$$

As for $\delta-0$, we obtain a constant pressure from the solvability conditions. The heat equations are similar as before except that the first order approximation of $f$ appears in the source and loss term.

\subsection{Numerical Results}

Some additional simulations have been performed to test other scaling relations between the two parameters $\varepsilon$ and $\delta$ numerically. All simulations used a fixed parameter (Knudsen number) $\varepsilon=0.01$ and 25.600 particles. The boundary conditions are given by $(3.22)$ with $T_{L}=T_{R}=1.0$ and $T_{L, i}=T_{R, i}=3.0$ and we used again the rigid-spherical molecule model. The different values for $\delta$ are $\delta=0.0,0.001\left(=\varepsilon^{2}\right), 0.01(=\varepsilon), 0.1(=\sqrt{\varepsilon}), 0.9(-1-\sqrt{\varepsilon}), 1.0$ 
In Fig. 3 the curves are nearly constant, since there is no energy exchange between the kinetic and internal degrees of freedom. Small deviations appear in Fig. 4, which corresponds to the macroscopic equations. The case $\delta=0.01$ (Figure 5) seems to be very interesting, since the curves just touch each other. Figs. 6-8 show that the kinetic and rotational temperature are equal in a domain between the walls. If we increase the $\delta$-value, then this domain becomes larger.
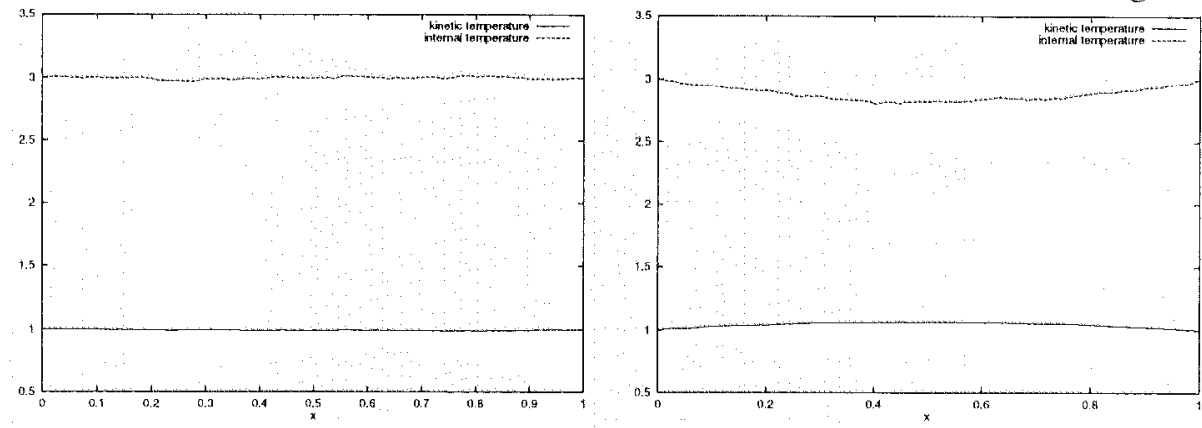

Figure $3 \quad \delta=0.0, \varepsilon=0.01$

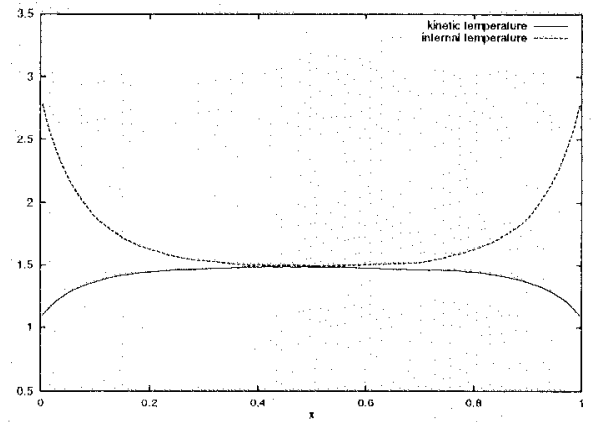

Figure $4 \delta=0.0001, \varepsilon=0.01$

Figure $5 \delta=0.01, \varepsilon=0.01$

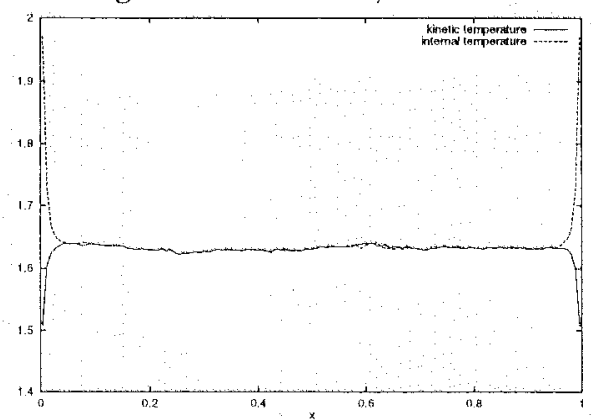

Figure $7 \quad \delta=0.9, \varepsilon=0.01$

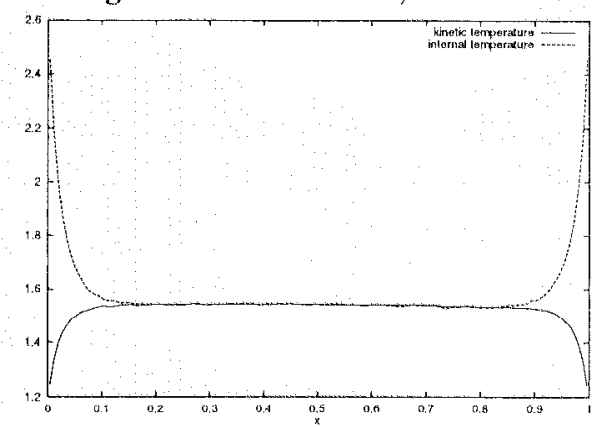

Figure $6 \quad \delta=0.1, \varepsilon=0.01$

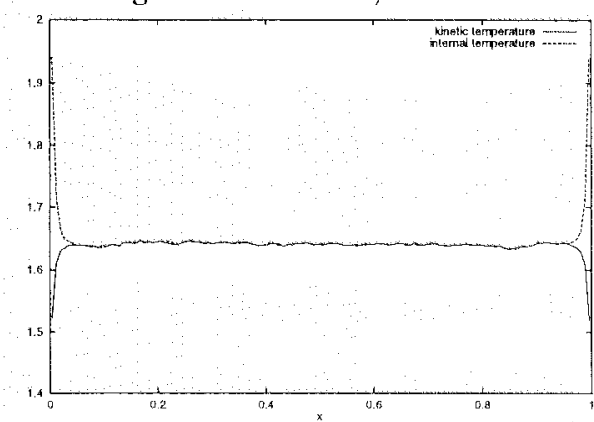

Figure $8 \quad \delta=1.0, \varepsilon=0.01$ 


\section{Navier-Stokes Limit by Levermore Expansion}

\subsection{Approximation by Moment Expansions}

The basic construction of an approximation in moments of the Boltzmann equation starts by choosing a linear space $\mathcal{E}_{m}$ of test functions in $(v, e)$ and integrating the Boltzmann equation at each spatial point $x$ against basis functions $m(v, e)$ of $\mathcal{E}_{m}$, which yields the system

$$
\partial_{t}\langle m f\rangle_{v, e}+\operatorname{div}\langle v \otimes m f\rangle_{v, e}=\langle m Q(f, f)\rangle_{v, e}
$$

For physical reasons, the choice $\mathcal{E}_{m}$ of test functions cannot be arbitrary: first, because the resulting system must satisfy fundamental conservation laws in mass, momentum and energy, this space must contain the functions $m=$ $\left\{1, v, v^{2}+e\right\}$, whose integral moments $\langle m f\rangle_{v, e}$ define the mass, velocity and total energy of the fluid. Second, the final system must be invariant under space translation and rotation, which means that the test space $\mathcal{E}_{m}$ itself must be stable under the action of any arbitrary translation and rotation.

The problem is, that the system (4.1) is not closed: equations above characterize the evolution of the averages $\langle m f\rangle_{v, e}$ by other averages $\langle v \otimes m f\rangle_{v, e}$ and the whole system can therefore only be solved, if one has additional information on the structure of the density function $f$. Different possibilities may be proposed at this level. Grad [5] builds the distribution function $f$ using thirteen independent Hermite polynomials in $v$ (for the monoatomic case). The resulting model predicts locally reasonable velocity jumps at solid boundaries, but it leads to a function $f$, which is not always positive. As a consequence, the notions of entropy and hyperbolicity are lost and the resulting boundary value problem is not well posed. A better choice, introduced in [13], which will be used in the following, is based on the Ansatz.

$$
F-F(\alpha)=\exp (\alpha \cdot m(v))
$$

with $m(v)=\left\{1, v, v \otimes v, v^{2} v, v^{4}\right\}$ for the monoatomic case and $m(v)=\left\{1, v, v \otimes v, I^{2},\left(v^{2}+2 I^{2}\right) v,\left(v^{2}+2 I^{2}\right)^{2}\right\}$ for the diatomic case. By construction, $F(\alpha)-\exp (\alpha \cdot m(v))$ is strictly positive. Moreover, it has nice entropy properties, since, as observed in [13], this distribution function is the formal solution of the entropy minimization problem

$$
F(\alpha)=\operatorname{argmin}_{f} \in x_{v}\langle f \ln f-f\rangle_{v, e}
$$


over the set $X_{U}$ of distributions with given moments $U$, i.e. the set $X_{U}=\left\{f:\langle m f\rangle_{v, e}=U\right\}$.

Substituting this ansatz in the moment equation (4.1), we obtain, that the kinetic moments $U_{\alpha}(\alpha)$, the kinetic fluxes $A_{\alpha}(\alpha)$ and the collision integrals $S(\alpha)$ with respect to the function $F(\alpha)=\exp (\alpha \cdot m)$ satisfy the system of partial differential equations with unknown $\alpha$ (or $U_{\alpha}$ ) in $\mathbb{R}^{14}$

$$
\partial_{t} U_{\alpha}(\alpha)+\operatorname{div} A_{\alpha}(\alpha)=S(\alpha)
$$

The weak formulation of the above conservative system (4.2) is simply obtained by multiplying the system by a test function $\phi(x)$ and integrating by parts in $x$, which yields

$-\int_{\Omega} \phi \partial_{t} U_{\alpha} \mathrm{d} x+\int_{\Omega} \partial_{x} \phi \cdot A_{\alpha} \mathrm{d} x-\int_{\partial \Omega} \phi \cdot\left\{A_{\alpha} \cdot n\right\} \mathrm{d} \gamma=-\int_{\Omega} \phi \cdot S_{\alpha} d x, \forall \phi(x)$.

The weak formulation (4.3) is certainly not well posed, because it lacks of boundary conditions specifying the boundary flux

$$
\left.A_{\alpha} \cdot n\right|_{\partial \Omega}:=\int_{v \cdot n} v \cdot n m(v) F(v) d v d e .
$$

To do this while respecting the underlying kinetic boundary conditions imposed on the distribution $f$, a simple and consistent choice is to split this boundary flux into ingoing and outgoing fluxes

$$
A_{\alpha} \cdot n=A_{\alpha}^{+}+A_{\alpha}^{-}, \quad A_{\alpha}^{+}:=\int_{v \cdot n \geq 0} v \cdot n m(v) F(v) d v d e
$$

with outgoing fluxes computed from the local value of the solution $F$ and ingoing fluxes from the imposed reflected values at any wall

$$
A_{\alpha}^{-}=\int_{v \cdot n \leq 0} v \cdot n m(v)\left[(1-k) \cdot M_{\rho, T_{p}, u}+k \int_{v^{\prime} \cdot n>0} \mathcal{R}\left(v, v^{\prime}\right) F\left(v^{\prime}\right) d v^{\prime}\right] d v d e
$$

or imposed incoming values at infinity

$$
A_{\alpha}^{-}=\int_{v \cdot n \leq 0}(v \cdot n) m(v) F_{i m p}(v) d v d e .
$$

The above notion of ingoing and outgoing fluxes is in fact related to the microscopic notion of ingoing or outgoing particles. The corresponding boundary condition amounts in fact to average the microscopic kinetic boundary condition against the basis test functions $m(v)$.

With this choice, the problem to solve is the weak nonlinear variational problem (4.3) with boundary conditions (4.4)-(4.5)-(4.6). 


\subsection{Relation to Navier-Stokes}

Easy algebraic manipulations transform the Levermore monoatomic system into five conservation laws (with $p=-\operatorname{Tr}(\sigma) / 3$ )

$$
\begin{gathered}
\frac{\partial \rho}{\partial t}+u_{k} \cdot \frac{\partial \rho}{\partial x_{k}}+\rho \frac{\partial u_{k}}{\partial x_{k}}=0, \\
\frac{\partial u_{i}}{\partial t}+u_{k} \cdot \frac{\partial u_{i}}{\partial x_{k}}-\frac{1}{\rho} \frac{\partial \sigma_{i k}}{\partial x_{k}}=0 \\
\frac{\partial p}{\partial t}+\frac{\partial u_{k} p}{\partial x_{k}}-\frac{2}{3} \sigma_{j k} \cdot \frac{\partial u_{j}}{\partial x_{k}}+\frac{2}{3} \frac{\partial q_{k}}{\partial x_{k}}=0
\end{gathered}
$$

and eight plus one differential constitutive laws in stress, heat vector and an additional moment $\mathcal{R}_{j j i k}=\left\langle|v|^{4} f\right\rangle_{v}$.

$$
\begin{array}{r}
\frac{\partial \sigma_{i j}}{\partial t}+\frac{\partial u_{k} \sigma_{i j}}{\partial x_{k}}+\sigma_{j k} \cdot \frac{\partial u_{i}}{\partial x_{k}}+\sigma_{i k} \cdot \frac{\partial u_{j}}{\partial x_{k}}-\frac{\partial Q_{i j k}}{\partial x_{k}}=\frac{1}{\epsilon}\left\langle v_{i} v_{j} Q(F, F)\right\rangle_{v} \\
2 \frac{\partial q_{i}}{\partial t}+2 \frac{\partial q_{i} u_{k}}{\partial x_{k}}+2 Q_{i j k} \frac{\partial u_{j}}{\partial x_{k}}+2 q_{k} \frac{\partial u_{i}}{\partial x_{k}} \\
+3 \frac{p}{\rho} \frac{\partial \sigma_{i k}}{\partial x_{k}}-2 \frac{\sigma_{i j}}{\rho} \frac{\partial \sigma_{j k}}{\partial x_{k}}+\frac{\partial \mathcal{R}_{j j i k}}{\partial x_{k}}=\frac{1}{\epsilon}\left\langle v^{2} v_{j} Q(F, F)\right\rangle_{v} \\
\frac{\partial \mathcal{R}_{i i j j}}{\partial t}+\frac{\partial \mathcal{R}_{i i j j} u_{k}}{\partial x_{k}}+4 \mathcal{R}_{j j i k} \frac{\partial u_{i}}{\partial x_{k}}+8 \frac{q_{i}}{\rho} \frac{\partial \sigma_{i k}}{\partial x_{k}}+\frac{\partial s_{k}}{\partial x_{k}}=\frac{1}{\epsilon}\left\langle v^{4} Q(F, F)\right\rangle_{v}
\end{array}
$$

These constitutive laws are no longer explicit : they involve auxiliary moments $Q$ and $\mathcal{R}_{j j i k}$ to be obtained by first computing the underlying distribution function $F^{14}$. But nevertheless, this nonconservative writing gives a direct way to recover Navier-Stokes equations from the Levermore equations. Indeed, let us perform a standard asymptotic expansion of the unknowns in terms of the mean free path $\varepsilon$, i.e. $F=F^{0}+\epsilon F^{0} \alpha^{1} \cdot m+O\left(\epsilon^{2}\right)$, where $F^{0}=\exp \left(\alpha^{0} \cdot m\right)$ and

$$
\begin{gathered}
\sigma_{i j}=\sigma_{i j}^{0}+\epsilon \sigma_{i j}^{1}+O\left(\epsilon^{2}\right) \\
Q_{i j k}=Q_{i j k}^{0}+\epsilon Q_{i j k}^{1}+O\left(\epsilon^{2}\right), \\
\mathcal{R}_{i i j k}=\mathcal{R}_{i i j k}^{0}+\epsilon \mathcal{R}_{i i j k}^{1}+O\left(\epsilon^{2}\right),
\end{gathered}
$$

with the normalization condition 


$$
\left\langle\Psi(v) \exp \left[\alpha^{0} \cdot m(v)\right]\right\rangle_{v}=\langle\Psi(v) \exp [\alpha \cdot m(v)]\rangle_{v}
$$

and $\Psi(v)=\left(1, v, v^{2}\right)^{t}$. At zero order, $\alpha^{0}$ must cancel the collision term, and therefore $F^{0}$ correspond to a Maxwellian, yielding

$$
\sigma_{i j}^{0}=-\rho R T \delta_{i j}, q_{i}^{0}=0, \mathcal{R}_{i i j j}^{0}=15 \rho R^{2} T^{2}
$$

At next order, because of (4.10), we have first $\left\langle\alpha^{1} \cdot m(v) F^{0}\right\rangle_{v}$ and after elimination of all time derivates by the conservation laws in a similar expansion of the constitutive laws $(4.7,4.9,4.9)$, on gets the following equations in $\alpha^{1}$,

$$
\begin{gathered}
\rho R T\left(\frac{\partial u_{j}}{\partial x_{i}}+\frac{\partial u_{i}}{\partial x_{j}}-\frac{2}{3} \delta_{i j} \frac{\partial u_{k}}{\partial x_{k}}\right)=\int_{v \in \mathbb{R}^{3}} 2 v_{i} v_{j} Q\left(F^{0} \alpha^{1} \cdot m, F^{0}\right) d v \\
5 \rho R T \frac{\partial R T}{\partial x_{i}}=\int_{v \in \mathbb{R}^{3}} 2 v^{2} v_{i} Q\left(F^{0} \alpha^{1} \cdot m, F^{0}\right) d v \\
0=\int_{v \in \mathbb{R}^{3}} 2 v^{4} Q\left(F^{0} \alpha^{1} \cdot m, F^{0}\right) d v
\end{gathered}
$$

By usual linearity and symmetry arguments ( even-odd symmetry of integrals in $v$, invariance, linearity in $\alpha^{1} \cdot m$ of the collision operator $\left.Q\left(F^{0} \alpha^{1} \cdot m, F^{0}\right)\right)$, the solution in $\alpha^{1} \cdot m$ is the standard Chapman-Enskog distribution

$$
\begin{gathered}
\epsilon \alpha^{1} \cdot m(v)=-\frac{2}{5} \frac{\kappa}{\rho(R T)^{2}}\left(\frac{c^{2}}{2 R T}-\frac{5}{2}\right) c \cdot \frac{\partial T}{\partial x_{i}} \\
-\frac{\mu}{\rho(R T)^{2}}\left(c \otimes c-\frac{1}{3} c^{2} I d\right):\left(\frac{1}{2}\left(\frac{\partial u}{\partial x}+\frac{\partial u^{T}}{\partial x}-\frac{2}{3} \operatorname{div}(u I d)\right)\right.
\end{gathered}
$$

yielding at first order in $\epsilon$ the Navier-Stokes constitutive laws

$$
\begin{gathered}
\epsilon \sigma_{i j}^{1}=-\epsilon\left\langle c_{i} c_{j} F^{0} \alpha^{1} \cdot m\right\rangle_{v}=\mu\left(\frac{\partial u_{j}}{\partial x_{i}}+\frac{\partial u_{i}}{\partial x_{j}}-\frac{2}{3} \delta_{i j} \frac{\partial u_{k}}{\partial x_{k}}\right) \\
\epsilon q_{i}^{1}=\epsilon\left\langle c^{2} c_{i} F^{0} \alpha^{1} \cdot m\right\rangle_{v}=-\kappa \frac{\partial T}{\partial x_{i}}
\end{gathered}
$$

and the additional law

$$
\epsilon \mathcal{R}_{i i j j}^{1}=\epsilon\left\langle c^{4} F^{0} \alpha^{1} \cdot m\right\rangle_{v}=0 .
$$

This means that any solution $\rho, u, T, \sigma, q$ of the Navier-Stokes equations is a first order approximate solution. 


$$
U_{\alpha}^{N S}=\left\{\begin{array}{l}
\rho \\
\rho u \\
\rho u \otimes u-\sigma \\
\rho u^{2} u-\operatorname{tr}(\sigma) u-2 \sigma \cdot u+2 q \\
\rho u^{4}-2 \operatorname{tr}(\sigma) u^{2}-4(\sigma \cdot u) \cdot u+8 q \cdot u+\frac{15 p^{2}}{\rho}
\end{array}\right.
$$

of the fourteen moment Levermore's equation, and conversely.

In particular, we get by this technique a new kinetic interpretation of a NavierStokes solution: its corresponding moments $U_{\alpha}^{N S}$ are an approximate solution of a consistent entropic hyperbolic problem and are moments of a positive distribution function $F_{N S}(v)=\exp (\alpha \cdot m(v))$ with coefficients $\alpha$ given by

$$
\langle m \exp (\alpha \cdot m(v))\rangle_{v}=U_{\alpha}^{N S}
$$

The difference with more standard models is, that now the definition of $F_{N S}$ is strictly positive, but no longer explicit and can only be obtained by solving a local entropy minimization problem.

\subsection{Numerical Coupling Strategy}

The additional advantage of the above kinetic interpretation is to lead to a simple multi-domain coupling strategy, which

1. solves the hyperbolic Levermore's model everywhere with semi-explicit schemes in time (collision implicit, transport terms explicit or linearly implicit) and finite volume in space,

$$
U_{\alpha, j}^{n+1}-U_{\alpha, j}^{n}=-\frac{\Delta t}{\operatorname{Vol} j} \sum_{i}\left(\mathcal{A}_{j, i}^{n}+\Delta t S\left(U_{\alpha, j}^{n+1}\right) .\right.
$$

2. gegenerate locally the Levermore's constitutive laws to their asymptotic analytic Navier Stokes limit in all cells, except where analytic residuals of Levermore differential constitutive equations are large.

In this strategy, the interface fluxes between different cells are then given by using a standard flux splitting scheme for interfaces between two Navier-Stokes cells and a kinetic reconstruction $F_{N S}$, which preserves positivity and entropy, for all other interfaces

$$
\mathcal{A}_{j, i}=\int_{t^{n}}^{t^{n+1}} \int_{\partial C_{j} \cup \partial C_{i}} v \cdot n F_{N S}^{n}\left(x-\left(t^{n+1}-t^{n}\right) v, v\right) d \gamma
$$

with $\mathcal{A}_{j i}^{n}=A_{j i}^{n_{1}+t}+A_{i j}^{n_{1}-}, A_{j i}^{n+t}=\int_{v, n_{j i} \geq 0} v \cdot n_{j i} m(v) F_{N S}^{n_{1}}\left(x_{j}, v\right) d v$ and $A_{j i}^{n_{1}-}$ computed from outside Levermore's distribution. The practical implementation of the above coupled scheme still remains rather technical because 
1. first, it involves nonlinear integrals in the velocity space, which can only be obtained by numerical quadrature [17], with a rule which must preserve local equilibria and must be used consistently everywhere (including at kinetic boundary conditions).

2. second, as observed in [7], for strong non-equilibrium states (inside strong shocks), there might be no coefficients $\alpha_{k}$ generating a given moment distribution $U_{k}$ in step 1.

The simulation below considers a two dimensional flow of a monoatomic gas over a flat plate at an angle of attack of $10^{\circ}$, Mach number 18.62 , Reynolds number $30687\left(\mathrm{~K} n=1 \cdot e^{-3}\right)$, temperature $194^{\circ} \mathrm{K}$ at infinity and $1000^{\circ} \mathrm{K}$ at wall. The viscosity is modeled by a hard sphere model, with $R=287$. The gas is modeled locally by a Navier-Stokes model away from the boundary and a Levermore's model next to the wall. We present iso-density lines (Fig. 9) and temperature, Mach values at three cross-sections at $x=.25, x=.55$ and $x=.85$ (Fig. 10), where the results are compared with those of a full Boltzinann solution.

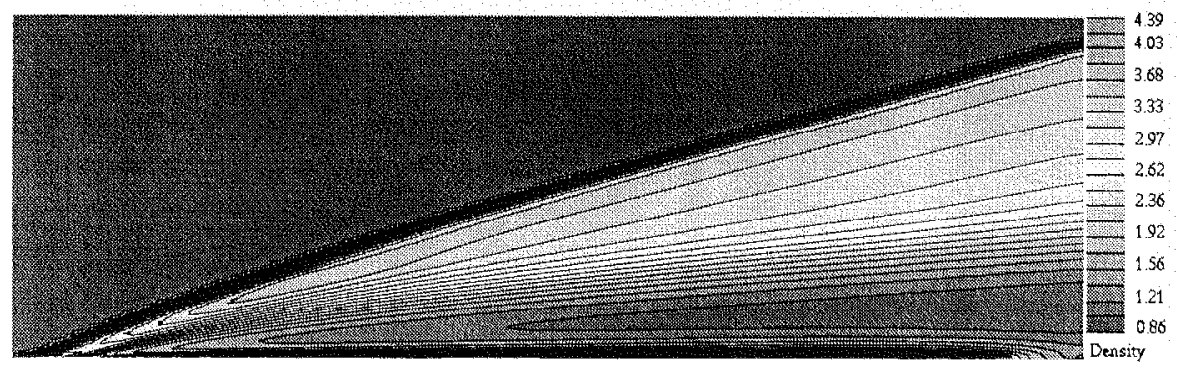

Figure 9 Iso-Density Values

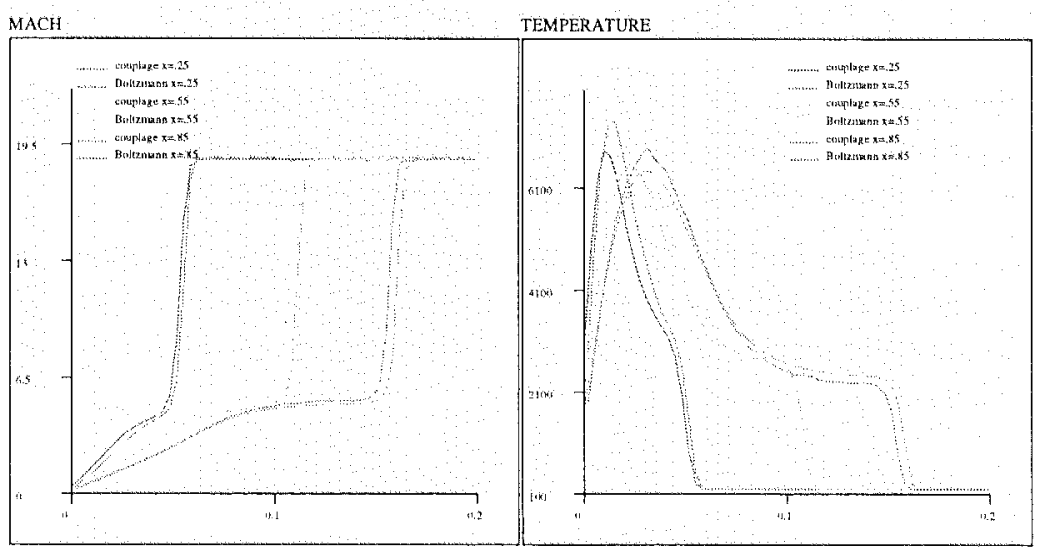

Figure 10 Mach and Temperature Values

Comparison with a Simulation for the Full Boltzmann Equation 


\section{Conclusion}

We presented some preliminary results on the derivation of fluid dynamic limits for the generalized Boltzmann equation with real gas effects. In particular, we studied the steady-state, one-dimensional Boltzmann equation with one additional internal energy modelled as a continuous variable. The different relaxation times are related using a small parameter $\delta$, which was later on related to the mean free path of the gas, assuming a fixed functional dependence between $\varepsilon$ and $\delta$. The resulting Navier-Stokes equations turned out to be enlarged by an additional temperature equation for the rotational temperature, with transport coefficients defined similar to the monoatomic case. Some numerical simulations were given to validate the asymptotic method to derive the fluid dynamic equations.

In the second part of the paper we present a new approach to define coupling procedures for the Boltzmann and Navier-Stokes equations, which are based on the 14-moments expansion of Levermore. In this expansion method, the density function remains strictly positive, which yields clear advantages with respect to the classical Grad's moment expansion. Moreover, we derived a new kinetic interpretation for the 14 -moments method of Levermore and give numerical simulation results on the resulting domain decomposition approach.

\section{Bibliography}

[1] Bourgat, J.-F., Desvillettes, L., Le Tallec, P. and Perthame, B.: Microreversible collisions for polyatomic gases and Boltzmann's theorem, European Journal of Mechanics, B/Fluids, 13, no 2, 237.254 (1994)

[2] Charrier, P., Dubroca, B. and Feugeas, J.L.. Etude numérique de modèles aux moments de Levermore en 2 dimensions, personal communication, 1996.

[3] Chapman, S. and Cowling, T.G.: The Mathematical Theory of Non-uniform Gases, Cambridge University Press (3th edition, 1970)

[4] Ferziger, J.H. and Kaper, H.G.: Mathematical theory of transport processes in gases, North-Holland (1972)

[5] Grad, H.: On the Kinetic Theory of Rarefied Gazes, Comm. Pure Appl. Math 2. pp 331-407, 1949

[6] Hash, D. and Hassan, A.. A Hybrid DSMC/Navier-Stokes Solver, AlAA 95-0410

[7] Junck, M.: About the domain of definition of Levermore's five moment sys tem, personal communteation; Tune 1997. 
[8] Kuščer, 1.: Dissociation and Recombination in an Inhomogeneous Gas, Physica A, 176, 542-556 (1991)

[9] Le Tallec, P. and Mallinger, F.: Coupling Boltzmann and Navier-Stokes equations by half fluxes, Journal of Computational Physics, 136, 51-67, 1997.

[10] Le Tallec,P and Perlat, J.-P. . Coupling Kinctic Models with Navier-Stokes equations, to appear in CFD Review, 1998

[11] Le Tallec, P. and Perlat, J.-P., "Asymptotic Kinetic Models for Transitional Flows ", Proceedings of the "International Conference on Numerical Modelling in Continuum Mechanics", M. Feistauer ed., Prague 1997.

[12] Le Tallec, P. and Perlat, J.-P. : Numerical Analysis of Levermore's Moment System, Rapport de recherche INRIA 3124, Mars 1997.

[13] Levermore, D.: Moment Closure Hierarchies for Kinetic Theories, Department of Mathematics, University of Arizona,submitted to the journal of statistical physics, May 1995.

[14] Levermore, D. and Morokoff, W.J.: The Gaussian Moment Closure for Gas Dynamics, SIAM J. on Applied Mathematics, submitted February 1996.

[15] Levermore, D.: Entropy Based Moment Closures for Kinetic Equations. Transport Theory and Statistical Physics, April 1996.

[16] Neunzert, H. and Struckmeier, J.: Particle Methods for the Boltzmann Equation, ACTA NUMERICA 1995, Cambridge (1995)

[17] Perlat, J.P.: Modélisation et Calcul parallèle d'une couche limite cinétique, Universit Pierre et Marie Curie, Paris VI, Janvier 1998.

[18] Sack, W.: Modellierung und Numerik für reaktive Strömungen in verdünnten Gasen, PhD thesis, University of Kaiserslautern, October 1995.

[19] Tiwari, S. and Klar, A. An Adaptive Domain Decomposition Procedure for Boltzmann and Euler Equations, to appear in Journal of Comp. \& Appl. Math 